\title{
Padres de niños con discapacidad. Relación y diferencias entre inteligencia emocional y resiliencia
}

\section{María Salazar-Farfán ${ }^{1}$}

RESUMEN: El objetivo fue determinar la relación entre la inteligencia emocional y la resiliencia en padres de familia de estudiantes con discapacidad de un centro educativo básico especial. Se realizó un estudio correlacional de corte transversal. Para el análisis estadístico se usó T-Student y ANOVA. Se empleó el Trait Meta-Mood Scale (TMMS-24) y la escala de resiliencia de Wagnild Young. Se encontró una proporción similar de escasa resiliencia (44.4\%) entre padres y madres de familia. Se evidencia una correlación significativa entre la inteligencia emocional y resiliencia con una relación directa entre los niveles de ambas variables. Aunque existe asociación entre el tipo de discapacidad y los niveles de ambas variables principales se manifiestan diferencias en su comportamiento respecto al tipo de discapacidad de los hijos. Se plantea la necesidad de intervenir con programas que potencien la inteligencia emocional y la resiliencia considerando estas diferencias.

PALABRAS ClAVE: Discapacidad; Inteligencia emocional; Resiliencia; Padres de familia.

Citar como: Salazar M. Padres de niños con discapacidad. Relación y diferencias entre inteligencia emocional y resiliencia. CASUS. 2017;2(3):156-162. 


\section{INTRODUCCIÓN}

La inteligencia emocional y la resiliencia son importantes para poder afrontar situaciones adversas o estresantes (1). Ambas ayudan a sobrellevar eventos no esperados como el nacimiento de un hijo con discapacidad (2). Este acontecimiento suele ser impactante y repercutirá a lo largo de toda la vida (2). Esta nueva situación cambia los esquemas de toda la familia, experimentado sentimientos y actitudes diferentes en función de las diversas informaciones recibidas $(2,3)$.

Diversos estudios señalan que ambas variables se encuentran asociadas a la mejora y transformación de problemas $(1,3,4)$. Asimismo al abordaje de crisis, errores en situaciones de aprendizaje y al crecimiento personal (4). Por ello es importante ofrecer a los padres de familia de hijos con discapacidad un espacio de expresión y aprendizaje de herramientas y estrategias para enfrentar de mejor manera la discapacidad y lo que ella implica (5).

Las familias suelen experimentar una serie de sentimientos negativos ante el nacimiento de un hijo con discapacidad (2). Esta situación, que es inesperada, suele diferir con las expectativas sobre el hijo esperado (2). La confusión y los sentimientos de aceptación, rechazo y culpabilidad se mezclan en los miembros de la familia, surgiendo constantemente preguntas sobre el porqué de esta situación $(2,4)$. A nivel nacional, según la primera encuesta sobre discapacidad (ENEDIS), el 5.2\% de la población presenta alguna discapacidad (6). Mientras que el $88.0 \%$ de personas con discapacidad no reciben tratamiento y/o terapia de rehabilitación, solo un $25.4 \%$ recibe tratamiento psicológico y $5.1 \%$ asisten a grupos de autoayuda junto a sus familias (6). En Lima metropolitana existen 77 Centros educativos básicos Especiales (CEBE), a quienes les corresponde encontrar la manera de hacer partícipe a las familias de la vida escolar de sus hijos y lograr su intervención activa. Es decir, una implicación responsable en el proceso educativo (7).

Lo anterior implica que deben aprender a comunicar sus requerimientos con precisión, solicitar ayuda, manejar la ansiedad y controlar su lenguaje $(2,3)$. Incluso, tienen necesidad de acomodar la forma de relación y convivir entre los diversos miembros de la familia (3). Asimismo, existen reportes que señalan que un alto nivel de resiliencia permite a los padres de familia de niños con discapacidad tener una mejor adaptación y responsabilidad en cuanto al cuidado y educación de sus hijos y también tener una mejor calidad de vida (8).

Existen antecedentes que coinciden en que la inteligencia emocional y la resiliencia están asociadas entre sí $(1,2,4,9)$. De esta manera, los estudios señalan que ante el nacimiento de un niño con discapacidad los patrones familiares se alteran, las actividades familiares tienen que organizarse de forma diferente para adaptarse a las necesidades especiales del hijo con discapacidad $(2,4,9)$. Muchas veces, los recursos financieros suelen gastarse en el hijo con discapacidad más que en los otros hijos, o en otras necesidades de la familia (10). Con el tiempo las reacciones de los padres van cambiando aunque siguen experimentando momentos de dolor, angustia y conflictos en el curso de la vida de su hijo $(4,9)$.

A pesar de la existencia de un alto porcentaje de estudiantes con discapacidad en Perú (6), existen pocos estudios que se orienten a establecer la asociación entre la inteligencia emocional y la resiliencia de sus padres. En el contexto nacional, se conoce poco sobre las diferencias respecto a los recursos emocionales de los padres entre los distintos tipos de discapacidad de sus hijos. La complejidad de abordaje de la discapacidad, por un lado, y la necesidad de acompañamiento de los padres por otro, ponen en evidencia que es necesario conocer con mayor precisión la relación entre la inteligencia emocional y la resiliencia considerando las diferencias entre los diversos tipos de discapacidad. De esta manera se propuso como objetivo explicar la relación entre la inteligencia emocional y resiliencia en padres de familia de un CEBE de Lima Norte.

\section{MATERIALES Y MÉTODOS}

Estudio de tipo correlacional de corte transversal. Se consideró una muestra de 140 padres de familia de alumnos de educación especial con discapacidad de un centro educativo básico especial de Lima Norte, seleccionados con un muestreo probabilístico.

Las variables principales fueron: inteligencia emocional y resiliencia. La inteligencia emocional se define como el conjunto de habilidades para percibir con precisión, valorar y expresar las emociones; para acceder y/o generar sentimientos cuando facilitan pensamientos; de comprender la emoción y el conocimiento emocional; para regular las emociones para promover crecimiento emocional e intelectual 
(11). Por otra parte, la resiliencia es definida como una característica de la personalidad que modera el efecto negativo del estrés y fomenta la adaptación, ello connota vigor o fibra emocional y se ha utilizado para describir a personas que muestran valentía y adaptabilidad ante los infortunios de la vida (12). La inteligencia emocional es una variable numérica cuantitativa discreta y se midió a través del Trait Meta-Mood Scale (TMMS-24). La misma comprende tres dimensiones: atención emocional, claridad emocional, reparación emocional y fue categorizada en tres niveles: bajo ( 0 a 24$)$, adecuado $(25$ a 35$)$ y excelente (mayor a 35) que corresponden al nivel de inteligencia emocional. La resiliencia es una variable numérica cuantitativa y se midió a través de la escala de resiliencia de Wagnild Young. Este inventario categoriza a la resiliencia en tres niveles: escasa (menor a 121), moderada (121 a 146) y mayor (147 a 175). Ambas pruebas se validaron, obteniéndose para Trait Meta-Mood Scale (TMMS-24) un valor de alfa de cronbach de 0.85 y para la escala de resiliencia de Wagnild Young 0.975.

Igualmente se consideraron las siguientes variables: sexo, variable dicotómica y tipo de discapacidad. Para la clasificación de las mismas se empleó el criterio utilizado por el Ministerio de educación peruano (13). Variable nominal (síndrome de Down, trastorno del espectro del autismo (TEA), discapacidad intelectual y discapacidad auditiva).

Para el análisis de los datos se utilizó el paquete estadístico STATA. A nivel descriptivo se consideraron frecuencias y porcentajes. Se empleó la prueba de T-Student y ANOVA en el análisis de las variables. Se consideró una significancia estadística menor a 0.05 . En la recolección de información se respetó la privacidad y el anonimato de los padres de familia.

\section{RESULTADOS}

Los resultados reportan que un $75.7 \%$ de los encuestados fueron mujeres, mientras que un $36.4 \%$ de los hijos de los padres de familia presentan el diagnóstico de síndrome de Down. Asimismo, el $60 \%$ del total presentó un nivel bajo en la dimensión atención emocional. Mientras que el $44.3 \%$ de la muestra reportó un nivel escaso de resiliencia. El resto de los datos se puede observar en la tabla 1.

Los hallazgos indican que entre padres y madres de familia no existe diferencia respecto a la escasa

\section{Tabla 1: Descripción de las variables en padres de familia}

\begin{tabular}{|c|c|c|}
\hline & $\mathrm{n}$ & $\%$ \\
\hline \multicolumn{3}{|l|}{ Sexo } \\
\hline Femenino & 106 & 75.7 \\
\hline Masculino & 34 & 24.3 \\
\hline \multicolumn{3}{|l|}{ Diagnóstico } \\
\hline Síndrome de Down & 51 & 36.4 \\
\hline TEA & 39 & 27.9 \\
\hline Discapacidad Intelectual & 25 & 17.9 \\
\hline Discapacidad Auditiva & 25 & 17.9 \\
\hline \multicolumn{3}{|l|}{ Atención emocional } \\
\hline Bajo & 84 & 60.0 \\
\hline Adecuado & 52 & 37.1 \\
\hline Excelente & 4 & 2.9 \\
\hline \multicolumn{3}{|l|}{ Claridad emocional } \\
\hline Bajo & 71 & 50.7 \\
\hline Adecuado & 59 & 42.1 \\
\hline Excelente & 10 & 7.2 \\
\hline \multicolumn{3}{|l|}{ Reparación emocional } \\
\hline Bajo & 59 & 42.1 \\
\hline Adecuado & 62 & 44.3 \\
\hline Excelente & 19 & 13.6 \\
\hline \multicolumn{3}{|l|}{ Resiliencia } \\
\hline Escasa & 62 & 44.3 \\
\hline Moderada & 31 & 22.1 \\
\hline Mayor & 47 & 33.6 \\
\hline
\end{tabular}

capacidad de resiliencia (44.4\%). Existe relación significativa entre la inteligencia emocional y la resiliencia $(p=0.00)$. De igual manera existe relación entre la discapacidad y la resiliencia $(\mathrm{p}=0.00)$. Los resultados señalan un $66 \%$ de padres con hijos con síndrome de Down con escasa resiliencia, mientras que un $56 \%$ de padres con hijos con discapacidad intelectual reportan un nivel mayor de resiliencia. Los resultados muestran un $41.1 \%$ de padres con un nivel excelente de resiliencia. También se observó que existe relación entre las dimensiones de inteligencia emocional y la resiliencia. Se reporta un mayor porcentaje de padres de familia con un nivel bajo en la dimensión atención emocional (61.0\%). El resto de los datos se puede observar en la tabla 2 .

En la tabla 3 se evidencia que no existe asociación significativa entre la inteligencia emocional y el sexo 
Tabla 2. Asociación entre la inteligencia emocional y resiliencia

\begin{tabular}{|c|c|c|c|c|}
\hline & \multicolumn{4}{|c|}{ Resiliencia } \\
\hline & Escasa & Moderada & Mayor & $\mathbf{p}$ \\
\hline & $\%$ & $\%$ & $\%$ & \\
\hline Sexo & & & & 0.39 \\
\hline Femenino & 44.4 & 24.5 & 31.1 & \\
\hline Masculino & 44.1 & 14.7 & 41.2 & \\
\hline Diagnóstico & & & & 0.00 \\
\hline $\begin{array}{l}\text { Síndrome de } \\
\text { Down }\end{array}$ & 66.7 & 23.5 & 9.8 & \\
\hline TEA & 17.9 & 30.8 & 51.3 & \\
\hline $\begin{array}{l}\text { Discapacidad } \\
\text { Intelectual }\end{array}$ & 36.0 & 8.0 & 56.0 & \\
\hline $\begin{array}{l}\text { Discapacidad } \\
\text { Auditiva }\end{array}$ & 48.0 & 20.0 & 32.0 & \\
\hline $\begin{array}{lc}\text { Inteligencia } & \text { media } \\
\text { emocional } & \text { (DS) }\end{array}$ & $\begin{array}{c}68.7 \\
(17.6)\end{array}$ & $\begin{array}{c}77.2 \\
(15.7)\end{array}$ & $\begin{array}{c}81.3 \\
(16.8)\end{array}$ & 0.00 \\
\hline $\begin{array}{l}\text { Atención } \\
\text { emocional }\end{array}$ & & & & 0.02 \\
\hline Bajo & 52.4 & 23.8 & 23.8 & \\
\hline Adecuado & 34.6 & 19.2 & 46.2 & \\
\hline Excelente & - & 25.0 & 75.0 & \\
\hline Claridad emocional & & & & 0.00 \\
\hline Bajo & 59.2 & 18.3 & 22.5 & \\
\hline Adecuado & 28.8 & 27.1 & 44.1 & \\
\hline Excelente & 30.0 & 20.0 & 50.0 & \\
\hline $\begin{array}{l}\text { Reparación } \\
\text { emocional }\end{array}$ & & & & 0.01 \\
\hline Bajo & 61.0 & 17.0 & 22.0 & \\
\hline Adecuado & 30.7 & 29.0 & 40.3 & \\
\hline Excelente & 36.8 & 15.8 & 47.4 & \\
\hline
\end{tabular}

de los encuestados. Los valores medios más bajos de inteligencia emocional corresponden a la dimensión atención emocional (media de las madres $=22.9$ ). Existe asociación significativa entre la inteligencia emocional y el tipo de discapacidad de los hijos de los encuestados. Los valores medios más bajos de inteligencia emocional corresponden a la dimensión atención emocional en los padres cuyos hijos presentan el diagnóstico de síndrome de Down (media
$=19.8)$. El resto de los datos se puede observar en la tabla 3 .

\section{DISCUSIÓN}

Los resultados revelan que la inteligencia emocional se asocia en modo directo a la resiliencia. Así también ambas variables se relacionan con el diagnóstico de discapacidad de los hijos de los encuestados. Aunque en los padres se manifiestan diferencias en el comportamiento de las variables principales respecto a la discapacidad de los hijos.

Se observó entre los encuestados la asociación significativa entre la inteligencia emocional y la resiliencia evidenciando que mayores puntuaciones de inteligencia emocional corresponden a mayores niveles de resiliencia. Este hallazgo es corroborado por otros estudios que señalan que además de existir relación también existe dependencia entre ambas variables $(14,15)$. Los resultados coinciden con la explicación encontrada en la evidencia científica ya que se atribuye a la inteligencia emocional un papel mediador que potencia la resiliencia $(1,2)$. Por esto ante situaciones vitales que requieren de un ajuste emocional favorecen las emociones positivas ante las desadaptadas (16). En este sentido, se debe considerar que las familias experimentan cambios cuando nace un nuevo miembro. Estas modificaciones igualmente se manifiestan con el nacimiento de un hijo con discapacidad (2). Sin embargo, en estos casos la familia necesita de mayor apoyo para desarrollar su capacidad para conocer y regular sus emociones $(1,4)$.

Existe asociación entre las dimensiones de la variable inteligencia emocional y los niveles de resiliencia. Se evidencia una relación directa ya que bajos niveles en las dimensiones de la inteligencia emocional corresponden a niveles inferiores de resiliencia y viceversa. Los resultados muestran que las personas que perciben, conocen y manejan sus emociones pueden gestionar mejor sus problemas emocionales $y$, por lo tanto, pueden vivenciar experiencias de mayor bienestar $(1,17)$. Tener un hijo con discapacidad involucra diversas situaciones de confusión, en las cuales se manifiestan sentimientos de aceptación, rechazo y culpabilidad $(4,5)$. Por tanto, es de esperar que aquellos padres de familia que no manejen adecuadamente las propias emociones presenten también un menor nivel de resiliencia $(1,4,5)$. Es decir, presentan niveles bajos los padres que no creen en su adecuada capacidad para regular estados emocionales negativos y prolongar los positivos. Así 
como los que se auto perciben con una inadecuada capacidad para presentar respuestas adaptativas ante situaciones adversas (4). equilibrio. Se necesita de una amplia gamas de intervenciones, según la necesidad, debido a que existe una ruptura del equilibrio que implica aceptar en la

Tabla 3. Asociación entre las variables inteligencia emocional con sexo y diagnóstico

\begin{tabular}{|c|c|c|c|c|c|c|c|c|}
\hline & $\begin{array}{l}\text { Atención } \\
\text { emocional } \\
\text { (media) }\end{array}$ & p-value & $\begin{array}{l}\text { Claridad } \\
\text { emocional } \\
\text { (media) }\end{array}$ & p-value & $\begin{array}{l}\text { Reparación de } \\
\text { emociones } \\
\text { (media) }\end{array}$ & p-value & $\begin{array}{c}\text { Inteligencia } \\
\text { emocional } \\
\text { (media) }\end{array}$ & p-value \\
\hline Sexo & & 0.22 & & 0.05 & & 0.03 & & 0.06 \\
\hline Femenino & 22.9 & & 24.8 & & 25.3 & & 73.1 & \\
\hline Masculino & 24.1 & & 27.3 & & 28.8 & & 80.2 & \\
\hline Diagnóstico & & 0.00 & & 0.00 & & 0.00 & & 0.00 \\
\hline $\begin{array}{l}\text { Síndrome de } \\
\text { Down }\end{array}$ & 19.8 & & 21.9 & & 22.8 & & 64.5 & \\
\hline TEA & 27.1 & & 30.1 & & 30.6 & & 87.7 & \\
\hline $\begin{array}{l}\text { Discapacidad } \\
\text { Intelectual }\end{array}$ & 25.1 & & 26.9 & & 27.8 & & 79.8 & \\
\hline $\begin{array}{l}\text { Discapacidad } \\
\text { Auditiva }\end{array}$ & 22.4 & & 23.6 & & 24.6 & & 70.6 & \\
\hline
\end{tabular}

Por otro lado, se observó que existe relación entre el tipo de discapacidad y la resiliencia. Los resultados señalan un $66 \%$ de padres con hijos con síndrome de Down con escasa resiliencia. Estos hallazgos han sido corroborados por otros estudios $(8,18)$. Una posible explicación podría relacionarse con los problemas a los que se enfrentan los padres de personas con el diagnóstico de síndrome de Down que van desde aspectos cognitivos (discapacidad intelectual) hasta emocionales y conductuales (19). Es frecuente en esta situación que afloren sentimientos de rechazo, miedo, impotencia, culpabilidad, o que no se sienta al hijo como propio, pues no es el que habían imaginado (5). Igualmente, que se produzca un auto cuestionamiento, que se generen conflictos en la pareja, entre otros problemas que hacen emerger dificultades a nivel emocional e influyen en la capacidad de moderar el efecto negativo del estrés y moderar la adaptación ante esta situación particular $(11,20)$.

Otra explicación podría relacionarse con la falta de programas para potenciar los factores protectores de resiliencia en padres con hijos con síndrome de Down. Se ha reportado que lo que afecta positiva o negativamente la vida de un niño con discapacidad, no solo depende de sus características, sino de la forma en que sus padres lo ven, lo tratan y de lo que hacen por él $(20,21)$. El nacimiento de un hijo trastoca el equilibrio existente en el sistema familiar y requiere del proceso hasta alcanzar un nuevo familia a un nuevo miembro con sus peculiaridades (22). Con este fin, probablemente, la familia tendrá que ir reconstruyendo su mundo de sentido, para dar significado y valor a un acontecimiento y una presencia al que el entorno sociocultural normalmente no se lo da (4).

Igualmente los hallazgos señalan que existe asociación significativa entre la inteligencia emocional y el diagnóstico de los hijos de los encuestados. Los valores medios más bajos de inteligencia emocional corresponden a la dimensión atención emocional en los padres cuyos hijos presentan el diagnóstico de síndrome de Down. Es importante mencionar que la atención emocional se refiere al grado en el que los individuos pueden identificar convenientemente sus propias emociones siendo el inicio del conocimiento a nivel emocional $(1,11)$. El mismo es importante para mejorar la adaptación positiva en relación a la necesidad educativa especial que presente un miembro de la familia $(2,22)$. De este modo los padres de familia con mayor atención a sus emociones pueden evidenciar un nivel más alto de malestar en su vida.

Entre las principales limitaciones del estudio se señala que no fueron incluidas variables que pueden estar relacionados con la inteligencia emocional y la resiliencia como la edad del hijo y el tipo de familia de proveniencia. A pesar de esta limitación los principales hallazgos concuerdan con estudios publicados. 


\section{CONCLUSIÓN}

Existe una relación directa entre las variables estudiadas evidenciando que mayores puntuaciones de inteligencia emocional corresponden a mayores niveles de resiliencia en los padres encuestados. Asimismo, el tipo de discapacidad de los hijos de los encuestados está asociado a la inteligencia emocional y la resiliencia. Sin embargo, existen diferencias respecto a los niveles de resiliencia reportados en relación a los tipos de discapacidad estudiadas.

En el ámbito familiar se recomienda seguir investigando sobre las diferencias respecto a los tipos de discapacidad. Finalmente, se plantea la necesidad de intervenir con los padres, a través de programas que potencien la inteligencia emocional. Los mismos deben informar la importancia del desarrollo emocional. El mismo permite tener una adaptación positiva a la discapacidad, un mejor desempeño en cuanto al cuidado y un mayor autocontrol. Estos aspectos tienen una repercusión positiva en la calidad de vida de las personas con discapacidad y sus familias. Dichos programas deben de hacer partícipe a las familias de la vida escolar de sus hijos e hijas y lograr su implicación activa. Es decir, responsable del proceso educativo y del desarrollo integral de la persona con discapacidad.

\section{REFERENCIAS BIBLIOGRÁFICAS}

1. Armstrong AR, Galligan RF, Critchley CR. Emotional intelligence and psychological resilience to negative life events. Personality and Individual Differences. 2011 Aug 31;51(3):331-6.

2. Heiman T. Parents of children with disabilities: Resilience, coping, and future expectations. Journal of developmental and physical disabilities. 2002 Jun 1;14(2):159-71.

3. Ministerio de educación del Perú: Guía del docente para orientar a las familias; 2013.

4. Lloyd TJ, Hastings R. Hope as a psychological resilience factor in mothers and fathers of children with intellectual disabilities. Journal of Intellectual Disability Research. 2009 Dec 1;53(12):957-68.

5. Marqués A. Análisis de la incidencia de Resiliencia en Padres y madres con hijos, que bajo el constructo de la diversidad, son etiquetados como discapacitados: Una propuesta de intervención en el ámbito socio familiar [tesis] Jaén: Universidad de Jaén; 2011.

6. Encuesta Nacional de discapacidad. Lima-Perú, 2012.

7. Ministerio de Educación: Directorio Educación básica especial: 2017.

8. Sac Mileny. Resiliencia en padres de niños con capacidades diferentes [tesis], Quetzaltenango: Universidad Rafael Landívar Guatemala; 2013.

9. Cejudo J, López-Delgado L y Rubio J. Inteligencia emocional y resiliencia: su influencia en la satisfacción con la vida en estudiantes universitarios. España: Universidad de Castilla-La Mancha; 2016.

10. Emerson E. Poverty and people with intellectual disabilities. Developmental Disabilities Research Reviews. 2007 Jan 1;13(2):107-13.

11. Mayer, J. y Salovey, P. ¿Qué es la inteligencia emocional? New York: Basic Books; 1997.

12. Wagnild GM, Young HM. Development and psychometric evaluation of the Resilience Scale. Journal of nursing measurement. 1993.

13. Ministerio de Educación. Manual de adaptaciones curriculares. Lima - Perú; 2007.

14. Portal R. Inteligencia emocional y adaptación en hijas que cuidan a su familiar con demencia [tesis]. Bogotá: Universidad de Granada; 2014.

15. Veloso, C. Relación entre inteligencia emocional, satisfacción vital, felicidad subjetiva y resiliencia en funcionarios de educación especial. [tesis]. Valdivia: Universidad Austral de Chile; 2013.

16. García del Castillo JA, G, Dias PC. Conceptualización teórica de la resiliencia psicosocial y su relación con la salud. Salud Drogas. 2016; 16(1):59-68.

17. Matthews G, Zeidner M, Roberts RD. Emotional intelligence 101. Springer Publishing Company; 2012.

18. Roque M, Tomasin G. Resiliencia materna, funcionamiento familiar y discapacidad intelectual de los hijos en un contexto marginado, Universidad Nacional Autónoma de México; 2012.

19. Sánchez M., Robles M. Respuesta a un programa de resiliencia aplicado a padres de niños con Síndrome de Down Universidad de Jaén, España; 2014.

20. Askoaga F. Trabajando con las familias de las personas con discapacidad. Instituto interamericano del niño: Organismo especializado de la OEA Montevideo; 2012.

21. Povee K, Roberts L, Bourke J, Leonard H. Family functioning in families with a child with Down syndrome: a mixed methods approach. Journal of Intellectual Disability Research. 2012 Oct 1;56(10):961-73.

22. King GA, Zwaigenbaum L, King S, Baxter D, Rosenbaum P, Bates A. A qualitative investigation of changes in the belief systems of families of children with autism or Down syndrome. Child: care, health and development. 2006 May 1;32(3):353-69. 
ISSN 2519-0652 CASUS.2017;2(3):156-162

\title{
Parents of children with disabilities. Relationship and differences between emotional intelligence and resilience
}

\begin{abstract}
The objective was to determine the relationship between emotional intelligence and resilience in parents of students with disabilities of a special basic education center. A crosssectional correlational study was carried out. For the statistical analysis T-Student and ANOVA was used. The Trait Meta-Mood Scale (TMMS-24) and the resilience scale of Wagnild Young were used. A similar proportion of low resilience $(44.4 \%)$ between fathers and mothers were found. There is a significant correlation between emotional intelligence and resilience with a direct relationship between the levels of both variables. Although there is an association between the type of disability and the levels of both main variables, there are differences in their behavior with respect to the type of disability of the children. There is a need to intervene with programs that enhance emotional intelligence and resilience considering these differences.
\end{abstract}

KEY WORDS: Disability; Emotional intelligence; Resilience; Parents. 